March 2008

\title{
Environmental Policy à la Carte: Letting Firms Choose their Regulation
}


The Author(s):

\section{Frank C. Krysiak}

University of Basel

Department of Economics

Petersgraben 51

4003 Basel

frank.krysiak@unibas.ch

\section{Iris Maria Oberauner}

University of Basel

Department of Economics

Petersgraben 51

4003 Basel

iris.oberauner@unibas.ch

A publication oft the Center of Business and Economics (WWZ), University of Basel.

(C) WWZ Forum 2008 and the author(s). Reproduction for other purposes than the personal use needs the permission of the author(s).

\section{Contact:}

WWZ Forum | Petersgraben 51 | CH-4003 Basel | forum-wwz@unibas.ch | www.wwz.unibas.ch 


\title{
Environmental Policy à la Carte: Letting Firms Choose their Regulation
}

\author{
Frank C. Krysiak* \\ Department of Economics, University of Basel, Switzerland \\ Iris Maria Oberauner \\ Department of Economics, University of Basel, Switzerland
}

\begin{abstract}
Environmental policy often has to be devised under informational constraints, like uncertainty and asymmetric information. We consider an environmental policy that aims at reducing the welfare losses caused by asymmetric information while being sufficiently simple for implementation. In this policy, firms can choose between being regulated with an emission tax or a permit market. This serves as a screening device; the firms reveal private information by choosing an instrument. We show that such a menu of policy options improves upon conventional environmental policy. Furthermore, the optimal policy is simple and thus easily implementable. The approach is also theoretically interesting, because the simultaneous use of price- and quantity-based instruments induces an asymmetry into the pricesversus-quantities decision compared to Weitzman's criterion. Especially, there can be an optimal pooling equilibrium where all firms choose the tax, but it is never optimal that all firms participate in permit trading.
\end{abstract}

Keywords: Environmental Policy, Asymmetric Information, Screening, Uncertainty, Prices-versus-Quantities JEL: Q58, D82, H23, D81, Q54

\footnotetext{
${ }^{*}$ Corresponding author; Frank Krysiak, University of Basel, Department of Economics, Petersgraben 51, 4003 Basel, Switzerland; e-mail: Frank.Krysiak@unibas.ch, fax: ++41 6126727 59, phone: ++41612673360
} 


\section{Introduction}

Traditionally, environmental policy is envisioned as a tool used by a governmental agency to regulate an industry's emissions. The agency levies a tax, sets a standard, or implements a permit market and the firms decide on production and abatement under the incentives provided by this policy.

Whereas this "top-down" type of policy performs well under perfect information, it is well-known that it can implement only a second-best allocation under informational constraints like asymmetric information or uncertainty [17]. Such constraints are ubiquitous in practice. Usually, firms have better knowledge concerning their technology than a regulation authority, so that there is asymmetric information, and the limited predictability of future factor prices induces uncertainty.

Numerous policy concepts have been devised to counter the effects of such incomplete information. Some of these concepts rely on ex-post adjustments of the policy, like automatic tax adjustments [3, 7], or adjustments to the number of permits as in [2, 7, 9]. Other concepts are based on a sophisticated instrument design that renders ex-post adjustments unnecessary, like a system of rental permits [1], the use of options in addition to permits [16], or the use of hybrid instruments [11].

These instruments are apt at reducing the social costs of uncertainty. Most of them can theoretically implement an ex-post efficient solution. However, this requires rather complex designs, like a large number of different options with differing strike prices, or it requires high levels of administrative flexibility, like instant tax adjustments or instant open market operations on a permit market. Thus in practice, these instruments will limit but not eliminate the detrimental effects of uncertainty.

Whenever the effects of uncertainty are not perfectly countered, the other type of informational constraints, that is, asymmetric information, will also reduce social welfare. Under uncertainty, the optimal choice of a regulation instrument depends on the technology of the regulated firms [17]. If this technology is private information, then the regulator might involuntarily use a suboptimal instrument and thereby reduce expected social welfare below the achievable level.

But in contrast to uncertainty, the welfare losses due to asymmetric information are not inevitable, even if only policy instruments with a limited complexity can be used. Whereas countering uncertainty requires to provide for all possible future states of nature, overcoming asymmetric information only demands to elicit already available information from the regulated firms. A potentially viable 
strategy for this is to decentralize a part of the regulatory decisions, that is, to let the regulated firms decide the type of regulation. In this way, decisions are made where the necessary information is available.

In other areas of economics, such concepts are common. For example, insurance companies are in a position comparable to an environmental regulator with regard to being subject to uncertainty and asymmetric information. To reduce the detrimental effects of asymmetric information, they frequently offer a range of insurance contracts, so that the insurance holder can decide which type of insurance policy is best for her. In this way, the insurance company elicits private information by partially delegating decisions to the party that has access to the necessary information. The situation is indeed similar to many environmental policy settings: Both the insurance holder and the insurance company lack information concerning future conditions that affect their profit, the insurance holder has private information that is needed to design an optimal insurance contract, and an optimal insurance policy requires to differentiate between different types of insurance holders.

But despite this similarity, comparable concepts are missing in environmental policy. There are contract designs that are used to solve problems with specific forms of asymmetric information, like non-point source pollution. 11 But asymmetric information with regard to technological characteristics is not addressed in this way, although this type of informational constraint is nearly ubiquitous in applications.

In this paper, we advance a policy concept where an important part of the policy decisions, that is the choice between a price- and a quantity-based regulation, is left to the regulated firms. The regulator announces a menu of policy options, in our case, an emission tax and permit trading, and each firm chooses its regulation from this menu. Thereby, both the firms and the regulator are subject to uncertainty at the time where the policy is chosen and the firms have private information concerning their technology.

This design is inspired by the current climate policy used in Switzerland, which consists of a carbon tax and an emissions trading scheme and allows the regulated firms to choose between these instruments. The firms pay the carbon tax but can get a refund if they commit to a prescribed emission reduction. If they make this reduction commitment, they receive tradable emission permits for the remaining emissions.

Using the model of [17], we show that such a policy menu can be designed so

\footnotetext{
${ }^{1}$ See , e.g., [6] or [13].
} 
that each firm voluntarily chooses the policy that is best for this firm from a social perspective. To design such a menu, only three ingredients are necessary: The regulator has to set an emission tax, has to issue emission permits, and has either to give a limited tax exemption or to auction some of the permits. Compared to the above mentioned policy designs, this concept is thus rather simple.

We provide a detailed characterization of the optimal design of this instrument and show that the optimal policy menu improves upon a single policy in terms of expected welfare. In addition, we analyze the firms' policy choices and show that, surprisingly, the firm that is indifferent between the two instruments will usually not have a technology that meets the Weitzman criterion for choosing between a price- and a quantity-based regulation. Rather, the optimal policy menu favors the emission tax; in many cases, the instruments should be designed so that a majority of firms will prefer the tax to emission trading. Furthermore, there can be cases where all firms should be induced to choose the tax, whereas there are virtually no cases in which all firms should be encouraged to participate in permit trading. Finally, we use a numerical example to highlight under which conditions our concept, which counters asymmetric information, is inferior or superior to the hybrid regulation of [11], which reduces the costs of uncertainty.

The paper is structured as follows. In the next section, we briefly review the relevant literature. In Section 3, we advance our model. In Section 4 , we charac-

terize the optimal policy. Section 5 provides a numerical example and Section 6 concludes.

\section{Review of the Literature}

Our study is related to the prices-versus-quantities literature and to the literature on screening on labor and insurance markets.

Initiated by [17], the prices-versus-quantities literature analyzes the choice between a price- and quantity-based environmental policy in a setting, where the marginal abatement costs of the regulated firms are uncertain at the time when the regulation is designed. As shown in [17] and [18], in the case of a single firm, a price-based instrument, like a tax, is preferable, if the marginal abatement cost function has a smaller slope than the marginal damage function, and a quantitybased instrument, like a permit market or a standard, is preferable in the opposite case. If there are several firms with distinct abatement cost functions, a weighted average of the slope of the firms' abatement costs has to be used for the comparison. So these studies show that under informational constraints, the preferability 
of regulation concepts depends on the firms' technologies.

Several studies have followed this line of analysis and advanced instruments that can improve upon a simple price or quantity regulation. Closest to our study is [11], who show that a hybrid regulation that consists of a permit market with a lower and an upper price limit (established by a subsidy and an emission tax, respectively), can achieve a higher expected welfare than each of these instruments alone. The price limits reduce the influence of uncertainty; whenever the permit price equals one of these limits, the aggregate emissions can deviate from the total number of permits and this flexibility increases social welfare.

Other instruments that can achieve similar effects are option trading, as analyzed in [16], where in addition to permits options for buying additional permits at ex-ante set prices are issued. Also, permits could be rented at prices that increase with the total number of permits [1], or the number of permits could be adjusted over time, as in [2, 9].

Our study deviates from these concepts in that we focus not on reducing the negative impact of uncertainty but rather on alleviating the detrimental effect of asymmetric information. To achieve this, we will use a setup where the policy is chosen and fixed before the uncertainty is resolved. In contrast, the above approaches rely all on providing some flexibility for ex-post adjustments. We will discuss the importance of this difference in the following section.

In this respect, our study comes close to the screening literature, where a set of contract options is considered as a screening device for a competitive insurance or labor market in cases in which information is asymmetrically distributed among agents, see [10, 12, 14]. The incomplete information problem arises, for example, with regard to the risk that individuals face, which is private information. The selfselection of a contract provides the insurance company with information about the individuals' risk exposure.

The screening literature differs from our approach in several aspects. In the screening literature, the contracts are designed to maximize the expected profit of the insurance company either under competition or in a monopoly. In contrast, we are concerned with minimizing the expected social costs of the regulation, which includes the expected profits of the regulated firms. Moreover, in the screening literature there is an "exit-option", that is, individuals are not obliged to buy an insurance. In our setup, firms have to choose a policy option.

The standard setting in the screening literature results in a separating equilibrium when two groups of risk types are to be considered and markets are competitive. The individuals select among partial and full insurance in order to maximize their utility. The case is somewhat different when individuals are represented by 
a continuum of risk types and the market is served by a monopolist [15]. The monopolist then does best by offering a continuum of contracts. Separation, noinsurance and pooling within a class of risk probability types may all be possible parts of the solution.

In our setting, both pooling and separating equilibria are also possible. However, it is not optimal to offer more than two policy options to the firms.

\section{The Model}

To analyze the benefits of letting firms choose their regulation, we consider an extension of the prices-versus-quantities model of [17]. A fixed number of firms emit a homogenous pollutant, which causes environmental damage. The firms can reduce their emissions by abatement. There are different types of firms that use different technologies and thus have different abatement cost functions. A regulator sets an environmental policy to reduce the total costs arising from environmental damage and the firms' abatement activities.

The regulation is subject to informational constraints. The regulator does not know which firm uses which technology, that is, there is asymmetric information with regard to a firm's type. Furthermore, the abatement costs are subject to random influences, like changing factor prices, demand-side shocks, or the breakdown of production equipment. Thus there is uncertainty both from the regulator's and from a firm's perspective.

Whereas the uncertainty is often an unavoidable problem, which could only be addressed by using sophisticated policy instruments that demand a high level of administrative flexibility [4], the detrimental effects of the asymmetric information can be reduced by using standard policy measures, albeit in an unconventional way. The idea is to provide individual firms with some choice concerning their regulation, so that a part of the regulation is decided by actors that have all the available information.

To this end we consider the following policy setup. The regulator offers the firms a choice between paying an emission tax and participating in an emission trading scheme. The firms have to commit to one policy option for several periods. The timing is shown in Figure 1. First, the regulator announces a menu of policy options, being subject to asymmetric information and uncertainty. Second, each firm commits to one of the policy options. Finally in each period, the actual abatement costs are observed and the firms make their abatement decisions under the chosen policy option. 


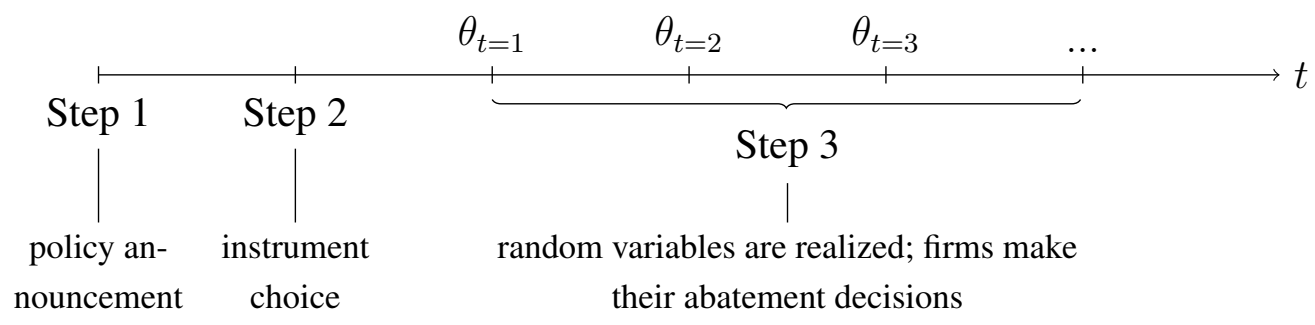

Figure 1: The timing of decisions in our model.

This setup models a setting, where abatement costs are subject to persistent uncertainty, which is often the case due to volatile factor prices. Firms have to choose their regulation knowing that they will commit for some time to this regulation and therefore they will choose according to their expected costs.

This differs from the setup of [11], where firms can choose in each period after observing their abatement costs whether to buy permits or pay a tax. This difference is crucial for gaining information about the firms' types: If firms can choose the regulation ex-post, their choices will be influenced both by their technology and by the realization of the random events. Thus their choice does not reveal private information about their technology. Indeed, in the setup of [11], all firms will choose the same policy option regardless of their technology. In contrast, if firms have to decide ex-ante about their preferred regulation, their choice depends only on their technology and thus provides useful information. We will work out the differences and their welfare implications more clearly in Section 4.

The choice of the two policy instruments, an emission tax and a permit market, is inspired by [17] and [18], who show that depending on the firms' abatement cost functions, either a price- or a quantity-based instruments should be used for regulation. Furthermore, this setup closely resembles the current climate policy in Switzerland, where firms are reimbursed for their payments of a carbon-based tax if they participate in a $\mathrm{CO}_{2}$-based emission trading scheme.

To analyze this policy, we use the following variant of Weitzman's model [17]. There is continuum of firms with mass one that have the following abatement cost function

$$
C(a, \beta, \theta):=(\alpha+\theta) a+\frac{a^{2}}{2 \beta},
$$

where $a$ denotes abatement, where $\theta$ depicts a random influence and where $\alpha \geq 0$ 
and $\beta>0$ describe a firm's technology ${ }^{2}$ We assume that the random influences are firm-specific but are positively correlated among firms with a correlation coefficient $\varrho \in] 0,1]$. The expected value of $\theta$ shall be zero and we denote its variance by $\sigma^{2}>0$. The values of the $\theta$ shall be intertemporally independent.

The firms differ with regard to their value of $\beta$. Given the results of [17] and [18], this is a natural choice. Of course in most real-world settings, firms' abatement costs will differ with regard to more parameters. But for sufficiently small random influences, the quadratic approximation used in (1) is adequate and as [17] has shown, only the second-order term is relevant for deciding between policy instruments $\sqrt{3}^{3}$ For analytical convenience, we assume that the parameter $\beta$ is uniformly distributed over $[\hat{\beta}-\Delta, \hat{\beta}+\Delta]$ with $\hat{\beta}>\Delta$ and $\Delta>0$.

The emissions of a firm exerting abatement effort $a$ are given by $e=\bar{e}-a$. The environmental damage caused by pollution $D(E)$ is described by the following quadratic function

$$
D(E)=\gamma E+\frac{E^{2}}{2 \delta},
$$

where $E$ denotes total pollution and where $\gamma \geq 0, \delta>0$ are constant parameters.

The policy consists of three measures. First, the regulator grants (freely) $z_{q}$ permits to each firm that participates in the emission trading scheme. Second, the regulator sets an emission tax $p_{p}$. Third, the regulator auctions additional permits or grants firms that have chosen the emission tax a partial tax exemption $z_{p}$.

Whereas the first two elements of the policy scheme are standard, the third one is less commonly discussed but crucial to the welfare effects of the policy. Usually, an emission tax has to be paid for all emitted units, whereas permits are often granted freely. Thus firms opting for emission trading receive a "subsidy" compared to the firms that pay the tax. This would result in an incentive to participate in emissions trading that is unrelated to a firm's technology. Auctioning a fraction of the permits or granting a partial tax exemption reduces and potentially reverses this incentive and thus allows to set a menu of policy options that guarantees that a firm chooses the type of regulation that is best from a social perspective.

\footnotetext{
${ }^{2}$ For notational simplicity, we use the reciprocal value of the second derivative of $C(a, \beta, \theta)$ as a technology parameter. Thus compared to the notation used in [17], we have $C^{\prime \prime}=1 / \beta$.

${ }^{3}$ Indeed, we could allow for different values of $\alpha$ among firms, but this would only result in a more complex notation without changing our results.
} 


\section{The Optimal Policy}

We derive the optimal policy by backward induction and thus begin with the firms' abatement decisions. Given that the random influences are intertemporally independent, it suffices to analyze a single period of the third stage of our model.

Consider a firm of type $\beta$ that opts to pay the emission tax. This firm will minimize its total cost, given the tax rate $p_{p}$ and the tax exemption $z_{p}$ :

$$
\min _{a \geq 0}(\alpha+\theta) a+\frac{a^{2}}{2 \beta}+p_{p}\left(\bar{e}-a-z_{p}\right) .
$$

This results in emissions 4

$$
e_{p}(\beta, \theta)=\bar{e}-\beta\left(p_{p}-\alpha-\theta\right),
$$

and expected costs

$$
\mathcal{E}\left(C_{p}(\beta)\right)=p_{p}\left(\bar{e}+\alpha \beta-z_{p}\right)-\frac{\beta}{2}\left(p_{p}^{2}+\alpha^{2}+\sigma^{2}\right) .
$$

As is apparent from (5), the firms' expected costs increase with the tax rate and decrease with the tax exemption. Also, the firm benefits from the cost uncertainty. With constant abatement, the firms' expected costs would be independent of $\sigma$, since the cost function is linear in the random variable. By optimally adjusting its abatement, the firm can improve upon this case and thereby decrease its expected costs. This decrease is proportional to the variance $\sigma^{2}$ of the random influence.

A firm that chooses to participate in the emissions trading scheme will minimize the costs

$$
\min _{a \geq 0}(\alpha+\theta) a+\frac{a^{2}}{2 \beta}+p_{q}\left(\bar{e}-a-z_{q}\right),
$$

where $z_{q}$ denotes the number of costlessly granted permits and where $p_{q}$ is the endogenously determined market clearing price for permits. The optimal emissions of such a firm are given by

$$
e_{q}(\beta, \theta)=\bar{e}-\beta\left(p_{q}-\alpha-\theta\right) .
$$

According to [17], we know that the permit market should contain firms with a high $\beta$ (and thus a low $C^{\prime \prime}$ in the notation of [17]) rather than those with a low $\beta$. Such an allocation of firms to the policy instruments is depicted in Figure 2 .

\footnotetext{
${ }^{4}$ Here, we have assumed that either the tax exemption is smaller than the emissions of the cleanest firm or that the tax exemption is granted in the form of a fixed subsidy that is not limited by actual emissions.
} 


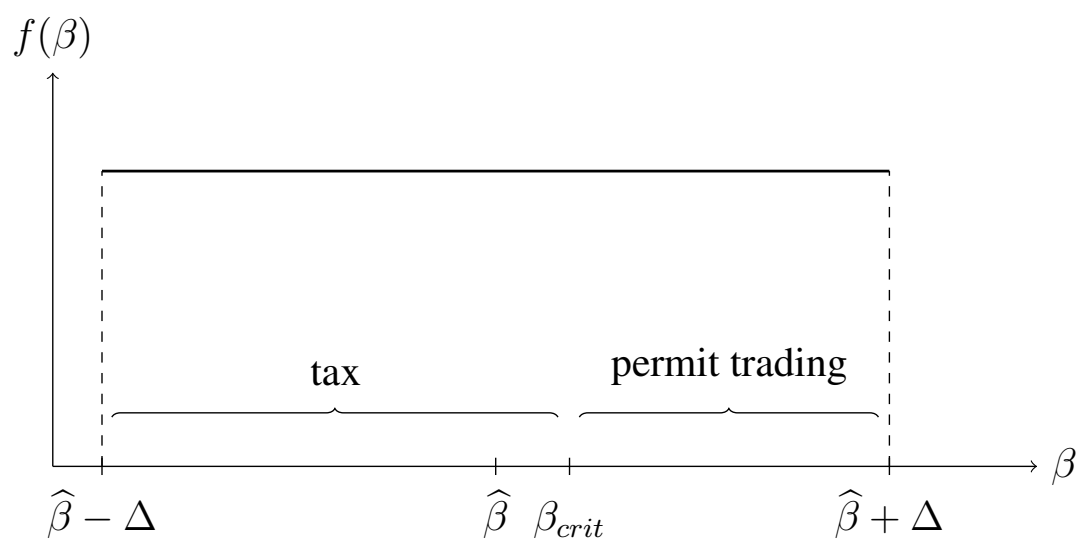

Figure 2: The firms' choice of policy instruments in dependency on their technology.

Assuming that the policy will be set in a way that all firms with $\beta \geq \beta_{\text {crit }}$ (with $\beta_{\text {crit }} \leq \hat{\beta}+\Delta$ ) will elect to participate in the emissions trading scheme, the market clearing condition can be written as

$$
\int_{\beta_{\text {crit }}}^{\hat{\beta}+\Delta} \frac{e_{q}(\beta, \theta)-z_{q}-z_{q}^{a u c}}{2 \Delta} d \beta=0,
$$

where $z_{q}^{a u c}$ denotes the number of permits per firm in the emission trading scheme that are auctioned.

The market clearing price for emission permits is thus

$$
p_{q}=\alpha+2 \frac{\bar{e}-z_{q}-z_{q}^{a u c}}{\beta_{\text {crit }}+\hat{\beta}+\Delta}+2 \frac{\theta_{p_{q}}}{\beta_{\text {crit }}+\hat{\beta}+\Delta},
$$

where $\theta_{p_{q}}$ denotes the changes of the permit price resulting from the individual cost changes $\theta$. Calculating $\theta_{p_{q}}$ shows that we have $\mathcal{E}\left(\theta_{p_{q}}\right)=0, \mathcal{E}\left(\theta_{p_{q}}^{2}\right)=$ $\left(\beta_{\text {crit }}+\hat{\beta}+\Delta\right)^{2} \varrho \sigma^{2} / 4$ and $\mathcal{E}\left(\theta_{p_{q}} \theta\right)=\left(\beta_{\text {crit }}+\hat{\beta}+\Delta\right) \varrho \sigma^{2} / 2$. The expected costs for a firm of type $\beta$ are therefore given by

$$
\begin{aligned}
\mathcal{E}\left(C_{q}(\beta)\right)= & \alpha\left(\bar{e}-z_{q}\right)-\frac{\beta}{2}(1-\varrho) \sigma^{2} \\
& +\frac{2\left(\bar{e}-z_{q}-z_{q}^{a u c}\right)\left(\beta z_{q}^{a u c}+\left(\bar{e}-z_{q}\right)\left(\beta_{c r i t}+\hat{\beta}+\Delta-\beta\right)\right)}{\left(\beta_{\text {crit }}+\hat{\beta}+\Delta\right)^{2}} .
\end{aligned}
$$


The firm's expected costs decrease both with the number of costlessly granted and the number of auctioned permits (albeit stronger with the former than the latter) and, again, the firm benefits from the cost uncertainty. However, in the case of emissions trading, the gain due to uncertainty is smaller. Due to $\varrho>0$, there is a correlation between the firms' random influences and thus between an individual firm's abatement costs and the permit price, which reduces the firm's abatement adjustments.

Given the expected costs under both policy options, we can now analyze a firm's choice from the menu of regulations. Comparing the expected costs under the tax regime (5) and under the permit trading scheme (10) shows that the difference between these costs depends linearly on $\beta$. Thus there can at most be a single type of firm (i.e., a single value of $\beta$ ) which is indifferent between the policy options; all other firms have strict preferences. We can identify this firm by equating the expected costs of the policy options and setting $\beta=\beta_{\text {crit }}$. In this way we get the following characterization of the indifferent firm.

$$
\begin{aligned}
p_{p} & \left(\bar{e}+\alpha \beta_{\text {crit }}-z_{p}\right)-\frac{\beta_{\text {crit }}}{2}\left(p_{p}^{2}+\alpha^{2}\right) \\
& -\left(\alpha\left(\bar{e}-z_{q}\right)+\frac{2\left(\bar{e}-z_{q}-z_{q}^{a u c}\right)\left(\beta_{c r i t} z_{q}^{a u c}+\left(\bar{e}-z_{q}\right)(\hat{\beta}+\Delta)\right)}{\left(\beta_{\text {crit }}+\hat{\beta}+\Delta\right)^{2}}\right) \\
& =\beta_{\text {crit }} \varrho \frac{\sigma^{2}}{2} .
\end{aligned}
$$

As this expression shows, a firm is indifferent between the instruments, if the gains from uncertainty under the tax regime are greater than the gains from uncertainty in the permit regime by an amount that compensates the difference in the total deterministic costs between the instruments. Since the gains from uncertainty are always greater in the tax than in the permit case, the total deterministic costs (i.e., the costs if future prices would be known with certainty) of paying the tax have to be greater for the indifferent firm than the total deterministic costs of participating in the permit trading scheme. So if, for example, the expected permit price should equal the tax rate and all permits are granted freely, then the tax exemption has to be smaller than the number of permits issued per firm.

To calculate the optimal policy, we also need information concerning the emis- 
sions, the resulting damage, and the tax revenue. The emissions are given by

$$
E=\frac{\left(z_{q}+z_{q}^{a u c}\right)\left(\hat{\beta}+\Delta-\beta_{c r i t}\right)}{2 \Delta}+\frac{\left(\beta_{\text {crit }}+\Delta-\hat{\beta}\right)\left(2 \bar{e}+2 \theta_{E}-\left(p_{p}-\alpha\right)\left(\beta_{c r i t}+\hat{\beta}-\Delta\right)\right)}{4 \Delta},
$$

where $\theta_{E}$ denotes the random variation of total emissions that is due to the random cost changes. Calculating $\theta_{E}$ shows that $\mathcal{E}\left(\theta_{E}\right)=0$ and $\mathcal{E}\left(\theta_{E}^{2}\right)=\left(\beta_{\text {crit }}+\hat{\beta}-\right.$ $\Delta)^{2} \varrho \sigma^{2} / 4$.

The expected environmental damage follows from substituting the total emissions (12) in the damage function (2) and calculating the expected value. Note that for $\beta_{\text {crit }}>\hat{\beta}-\Delta$, that is, whenever there are firms that choose the tax, the emissions include a random component. By the strict convexity of the damage function (2), this random component increases the expected damage. Thus as in [17], using a price-based regulation induces volatility in the aggregate emissions, which incurs social costs.

The expected tax revenue $\mathcal{E}(T)$ can be calculated as

$$
\mathcal{E}(T)=p_{p}\left(\beta_{\text {crit }}+\Delta-\hat{\beta}\right)\left(\frac{2 \bar{e}-2 z_{p}-\left(p_{p}-\alpha\right)\left(\beta_{c r i t}+\hat{\beta}-\Delta\right)}{4 \Delta}\right) .
$$

As usual, the expected tax revenue in dependency on the tax rate $p_{p}$ has the form of a Laffer curve, since increasing tax rates induce more abatement activity and thus eventually reduce the tax revenue.

Given the above information, the environmental policy is chosen to minimize social costs, given the firms' choice of regulation instrument, that is, the regulator solves

$\min _{p_{p}, z_{p}, z_{q}, z_{q}^{a u c} \geq 0} \int_{\hat{\beta}-\Delta}^{\beta_{\text {crit }}} \frac{\mathcal{E}\left(C_{p}(\beta)\right)}{2 \Delta} d \beta+\int_{\beta_{\text {crit }}}^{\hat{\beta}+\Delta} \frac{\mathcal{E}\left(C_{q}(\beta)\right)-p_{q} z_{q}^{a u c}}{2 \Delta} d \beta+\mathcal{E}(D(E))-\mathcal{E}(T)$,

s.t. (11).

The following proposition characterizes the solution of this optimization problem. 
Proposition 1. The optimal menu of policies is given by

$$
\begin{aligned}
p_{p} & =\frac{\bar{e}+\alpha \hat{\beta}+\gamma \delta}{\hat{\beta}+\delta}, \\
z_{q}+z_{q}^{a u c} & =\frac{\delta(\alpha-\gamma)\left(\beta_{c r i t}^{*}+\Delta+\hat{\beta}\right)+\bar{e}\left(2 \delta-\beta_{c r i t}^{*}-\Delta+\hat{\beta}\right)}{2(\hat{\beta}+\delta)} \\
z_{p} & =z_{q}-\varrho \sigma^{2} \frac{\beta_{c r i t}^{*}(\hat{\beta}+\delta)}{2(\bar{e}+\alpha \hat{\beta}+\gamma \delta)}
\end{aligned}
$$

Given this policy, the indifferent firm is characterized by

$$
\beta_{c r i t}^{*}= \begin{cases}\sqrt{(\hat{\beta}-\Delta)^{2}+2 \Delta \delta}, & \text { for } \delta \leq 2 \hat{\beta} \\ \hat{\beta}+\Delta, & \text { otherwise }\end{cases}
$$

All firms with $\beta<\beta_{\text {crit }}^{*}$ will opt for the emission tax, whereas all firms with $\beta>\beta_{\text {crit }}^{*}$ will participate in the emission trading scheme.

Proof. Calculating the derivatives of the expected social costs specified in (14) with respect to $p_{p}, z_{q}, z_{q}^{a u c}$ leads to a linear equation system with rank two and the solutions (15)-(16). The expected social costs are independent of $z_{p}$ and (11) is an implicit surjective mapping of $z_{p} \in \mathbb{R}$ to $\beta_{\text {crit }} \in[\hat{\beta}-\Delta, \hat{\beta}+\Delta]$. Thus we can optimize (14) with respect to $\beta_{\text {crit }}$ instead of $z_{p}$. This optimization yields three solutions, from which only $(18)$ can possibly fall into the range $[\hat{\beta}-\Delta, \hat{\beta}+\Delta]$, which is the case whenever $\delta \leq 2 \hat{\beta}$.

If $\delta \leq 2 \hat{\beta}$, then the Hessian of the expected social costs with regard to $p_{p}, z_{q}, \beta_{\text {crit }}$ is locally positive definite at $(15),(16)$, and $(18)$, so that these values indeed induce minimal expected social costs. If $\delta>2 \hat{\beta}$, then we have a boundary optimum at $\beta_{\text {crit }}=\hat{\beta}+\Delta$, because the expected social costs are strictly decreasing in $\beta_{\text {crit }}$ on $[\hat{\beta}-\Delta, \hat{\beta}+\Delta]$ in this case.

Finally, (17) follows from solving Eq. (11) under $(15)$ and $(16)$. That all firms with $\beta<\beta_{\text {crit }}^{*}$ choose the tax, whereas all firms with $\beta>\beta_{\text {crit }}^{*}$ will choose emission trading follows directly from comparing the firms' expected costs for both instruments under (15)-(18).

Proposition 1 provides a full characterization of the optimal policy. It shows that of the four policy variables $p_{p}, z_{p}, z_{q}, z_{q}^{a u c}$, three are needed to achieve the 
best feasible solution. This is intuitive; for each instrument, one policy variable is needed to set average emissions, and an additional policy variable is necessary to induce the socially desirable self-allocation of the firms to the policy instruments.

The optimal policy menu has several interesting characteristics, which we derive in the following corollaries.

Corollary 1. The tax rate in the optimal policy menu is the same tax that would be optimal if only an emission tax is used. Furthermore, the expected permit price equals the tax rate in the optimal policy menu.

Proof. Solving the regulators optimization problem for $\beta_{\text {crit }}=\hat{\beta}+\Delta$ yields (15) as a unique solution. Substituting (15)-(18) into (9) and calculating the expected permit price yields the r.h.s. of (15).

So, the optimal policy menu is very simple. It consists of an emission tax whose rate should be set as if the tax was the only implemented instrument. Then a sufficient number of permits is issued to assure that the expected permit price equals this tax rate.

This result is intuitive. Supplying permits so that the expected permit price equals the tax rate assures that the expected marginal abatement costs are the same for all firms, regardless of the instrument that they choose. This minimizes the total expected abatement costs for a given level of expected emissions. Since the expected marginal abatement costs are the same for all firms, the tax rate (and thus the expected permit price) can be chosen to equalize the expected marginal damage of emissions with the expected marginal abatement costs, which is a standard requirement for a second-best policy under cost uncertainty.

These two instruments suffice to assure an optimal allocation of emissions and abatement efforts. What remains is to assure an optimal allocation of the risk of cost changes and the possibly resulting emission volatility between firms and the society. As discussed above, a firm benefits from being able to adjust its abatement efforts to its actual costs. However, under a tax regime, where this is best possible, these adjustments lead to variations of the total emissions, which increase the expected damage. The relative strength of these effects depends on the technology of the firm. An optimal policy menu thus has to assure that those firms whose cost decrease due to better adjustment possibilities are greater than the increase in the expected damage due to having more volatile emissions choose the tax, whereas the other firms choose permit trading. The remaining policy variables $\left(z_{p}, z_{q}^{a u c}\right)$ can be used to this end. 
Corollary 2. Define

$$
\omega:=\frac{\delta(\alpha-\gamma)\left(\beta_{c r i t}^{*}+\Delta+\hat{\beta}\right)+\bar{e}\left(2 \delta+\hat{\beta}-\Delta-\beta_{c r i t}^{*}\right)}{2(\hat{\beta}+\delta)}-\varrho \sigma^{2} \frac{\beta_{c r i t}^{*}(\hat{\beta}+\delta)}{2(\bar{e}+\alpha \hat{\beta}+\gamma \delta)} .
$$

a) For every $z_{p} \in[0, \omega]$ and for every $z_{q}^{a u c} \in[0, \omega]$ a corresponding $z_{q}^{a u c}$ or $z_{p}$ can be found, so that the optimal policy menu specified in Proposition 1 can be achieved.

b) The tax exemption is always strictly smaller than the number offreely granted permits per firm.

c) It is never optimal to auction all permits.

Proof. By (17), $z_{q}$ is increasing in $z_{p}$. Furthermore, by (16), $z_{q}^{a u c}$ is decreasing in $z_{q}$. So the minimal feasible value of $z_{p}$, which is $z_{p}=0$, sets an upper bound for $z_{q}^{a u c}$ and the minimal feasible value of $z_{q}^{a u c}$ sets an upper boundary for $z_{p}$. Calculating these upper boundaries from (16) and (17) shows that both equal $\omega$ as defined in (19). Given the linearity of (16) and (17) in $z_{p}, z_{q}$, and $z_{q}^{a u c}$, the solution of (15)-(18) yields feasible values for all choices of $z_{p}$ or $z_{q}^{a u c}$ within the boundaries indicated in statement a).

Statement b) follows from (17), because the second term on the r.h.s. is strictly positive. Statement c) follows from setting $z_{p}=0$, which, by the above argument, leads to the minimal number of freely granted permits. According to (17), this minimal number is strictly greater than zero.

So as indicated in the above discussion, the optimal policy leaves one degree of freedom. Especially, it is possible to have no tax exemption, in which case an appropriate number of permits have to be auctioned, or to grant all permits freely, in which case an appropriate tax exemption is necessary. But it is never optimal to auction all permits or to grant a tax exemption that is greater or equal than the number of freely issued permits; both policy designs would lead to a too strong incentive for choosing the tax.

Up to now, we have characterized the optimal choice of the policy variables. What remains is to analyze the implicit allocation of firms to the price-based and the quantity-based regulation. The results of [17] and [18] suggest that firms with a low $\beta$ (and thus a high $C^{\prime \prime}$ in the notation of [17]) should be regulated with a tax and those with a high $\beta$ should participate in permit trading. The threshold for the instrument choice would be $\beta=\delta$. Indeed, calculating the expected social costs 
in our model for the cases where only an emission tax or only a permit market is used, shows that the former case dominates the latter with regard to expected social welfare whenever $\beta<\delta$, and the latter case dominates the former whenever $\beta>\delta$. So it would seem prudent to assume that an optimal policy menu is characterized by a similar symmetry between price- and quantity-based instruments and that the firm that is indifferent between optimally designed instruments should have a technology with $\beta=\delta$.

However, the following proposition shows that this is not true. There is a strong asymmetry between the price-based and the quantity-based part of an optimal policy menu.

Proposition 2. For $\delta>0$, it is always optimal that some firms choose the emission tax. In contrast, in an optimal policy menu there is only participation in the emissions trading scheme, if $\delta<2 \hat{\beta}$.

The indifferent firm has a technology with $\beta>\delta$, if $\delta<\Delta+\sqrt{(\hat{\beta}-\Delta)^{2}+\Delta^{2}}$, and it has a technology with $\beta<\delta$, whenever $\delta>\Delta+\sqrt{(\hat{\beta}-\Delta)^{2}+\Delta^{2}}$.

Proof. By (18), we have $\beta_{\text {crit }}^{*}=\hat{\beta}-\Delta$ (which is the permits only case) only for $\delta=0$, but we have $\beta_{\text {crit }}^{*}=\hat{\beta}+\Delta$ (the tax only case), whenever $\delta \geq 2 \hat{\beta}$. The further assertions follow from $(18)$, which shows that $\beta_{\text {crit }}^{*}$ is strictly monotonous increasing in $\delta$ with $\beta_{c r i t}^{*}=\delta$ for $\delta=\Delta+\sqrt{(\hat{\beta}-\Delta)^{2}+\Delta^{2}}$.

Thus the tax should always be chosen by some firms, if the marginal damage function is not completely inelastic. In contrast, there is an easily met condition under which the permit trading scheme should not be used at all. Whenever the slope of the marginal damage function is less than half of that of the marginal abatement cost function of the average firm, all firms should choose the emission tax. In the language of the screening literature, there can be a pooling equilibrium where all firms choose the tax, but a pooling equilibrium where all firms participate in permit trading is usually not optimal.

The reason for this asymmetry is the following. Consider the case, where the instruments are designed so that all firms choose permit trading. If we redesign the policy menu, so that the firms with the lowest value of $\beta$ (i.e., $\beta=\hat{\beta}-\Delta$ ) choose instead the emission tax, then the total expected abatement costs will be reduced. By Proposition 1, the tax will equal the average permit price, so that no distortions arise, and the firms that choose the tax will have larger gains due to being able to adjust their abatement efforts more strongly to their actual abatement cost. Indeed, 
the marginal change in total expected abatement costs due to a marginal increase of $\beta_{\text {crit }}$ at some value $\beta_{\text {crit }}^{\times}$is

$$
\left.\frac{\partial \mathcal{E}\left(C_{t o t}\right)}{\partial \beta_{c r i t}}\right|_{\beta_{c r i t}=\beta_{c r i t}^{\times}}=-\beta_{c r i t}^{\times} \frac{\varrho \sigma^{2}}{4 \Delta} .
$$

For $\beta_{\text {crit }}^{\times}=\hat{\beta}-\Delta$, this marginal effect is strictly negative.

The total expected damage will also change. By Proposition 1, both instruments induce the same expected emissions for a given firm type. But increasing $\beta_{\text {crit }}$ implies that emissions become more volatile, which, due to the strict convexity of the damage function, leads to a higher expected damage. However, if the change occurs at a low level of $\beta_{\text {crit }}$, only a small fraction of the emissions are volatile, so that the random changes are small compared to the total emissions. Indeed, the marginal effect of increasing $\beta_{\text {crit }}$ on the expected damage is given by

$$
\left.\frac{\partial \mathcal{E}(D(E))}{\partial \beta_{\text {crit }}}\right|_{\beta_{\text {crit }}=\beta_{\text {crit }}^{\times}}=\beta_{\text {crit }}^{\times}\left(\beta_{\text {crit }}^{\times^{2}}-(\hat{\beta}-\Delta)^{2}\right) \frac{\varrho \sigma^{2}}{8 \Delta^{2} \delta} .
$$

So if all firms choose permit trading $\left(\beta_{c r i t}^{\times}=\hat{\beta}-\Delta\right)$, then the marginal increase in expected damage due to inducing the firms with the lowest $\beta$ to choose instead the emission tax is zero, whenever $\delta>0$. In words: If only a tiny fraction of firms are regulated with the emission tax, the volatility of emissions is negligible compared to the total emissions and thus does not induce higher expected damage (the exception is the case $\delta \rightarrow 0$, where any variation of emissions leads to extreme damage increases).

Thus increasing $\beta_{\text {crit }}$ slightly from its minimal value induces gains but no costs and is therefore optimal.

In contrast, if we start with all firms choosing the emission $\operatorname{tax}\left(\beta_{\text {crit }}=\hat{\beta}+\right.$ $\Delta)$ and reduce $\beta_{\text {crit }}$, then this will increase the total expected abatement costs, because some firms have less adjustment possibilities. Also, it will reduce the expected damage, because the volatility of emissions decreases. Since none of these marginal effects is zero, we have to compare their magnitude. Eqs. (20) and (21), evaluated for $\beta_{\text {crit }}=\hat{\beta}+\Delta$, show that the abatement cost increasing effect is smaller than the damage reducing effect only if $\delta<2 \hat{\beta}$. Thus only under this condition, it is always optimal to induce some firms to participate in permit trading.

So the difference to the Weitzman setup is that the negative effect of having some firms under a price-based regulation is attenuated if other firms are regulated 


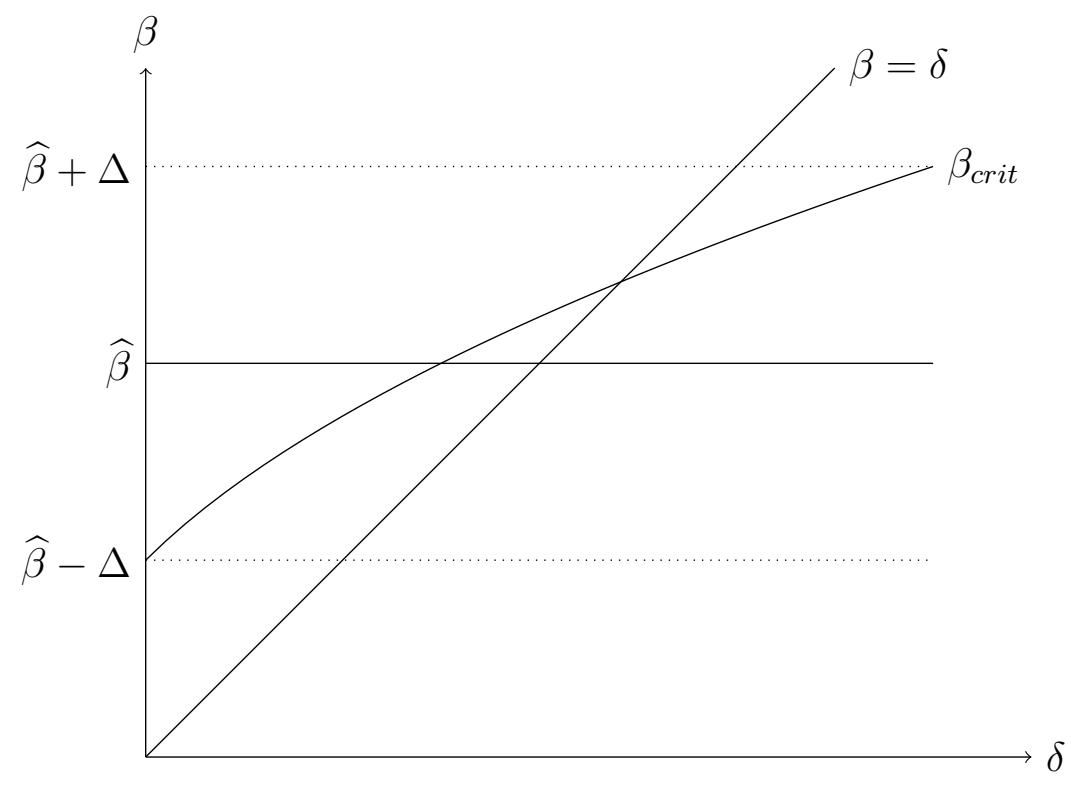

Figure 3: The indifferent firm in an optimal policy menu compared to the Weitzman criterion in dependency on $\delta$.

on the basis of quantities, because this negative effect arises from an aggregate, that is, the volatility of total emissions. In contrast, the negative effect of regulating firms with a quantity-based regulation is not reduced by the presence of priceregulated firms, because this effect is an individual effect, that is, the increase in individual abatement costs. Consequently, the simultaneous use of price- and quantity-based instruments renders the price-based part more favorable compared to the quantity-based part.

The second part of Proposition 2 emphasizes this point. We have depicted this in Figure 3. This figure shows that if we consider the same range of $\delta$ as we use for $\beta$, then for the majority of points, the optimal policy menu will induce more firms to choose the emission tax than the Weitzman criterion would suggest. That is, if there is a separating equilibrium, than this equilibrium is usually asymmetric in the sense that there are more firms that choose taxes than there are firms that choose permit trading.

The figure also shows that even if $\delta$ lies outside the range of $\beta$, it can be optimal to use a technology mix. This is always the case for $\delta<\hat{\beta}-\Delta$, so that we should use taxes and permit trading although all firms have a technology with 
$\beta>\delta$. It can also be the case for $\delta>\hat{\beta}+\Delta$, if $\Delta<\hat{\beta}$. In such cases, it is optimal to use both instruments, although all firms have a technology with $\beta<\delta$.

This shows that offering firms the opportunity to choose their regulation has welfare benefits for a considerable range of scenarios. Our concept encompasses the use of a single instrument policy as in [17]. But as Figure 3 shows, this is not optimal in many cases. Thus granting firms choices among policy options is often welfare increasing.

Given this point, an interesting question is whether the policy menu should be enlarged. Would it be beneficial to separate the firms into more than two groups? The answer to this question is negative; providing more than two policy options cannot be optimal. The reason is that as [17] has shown, price- and quantity-based policies differ only with regard to two effects: The increase in environmental damage due to the volatile emissions in a price-based regulation, and the increase in the firms' expected costs due to the reduced adjustment options in a quantitybased regulation. The relative importance of these effects depends monotonically on the difference between the curvature of the cost and the curvature of the damage function. But this implies that there can exist at most a single firm type at which the preferability of regulating a given firm with a price-based or a quantity-based policy switches. 5

Also, it is not reasonable to include other instruments, like standards, in the policy menu, because these induce higher expected abatement costs than tradable permits and have no benefits in terms of reducing the expected damage compared to a permit market.

Finally, we compare our approach to the hybrid regulation scheme of [11]. Our approach requires the firms to commit ex-ante to a regulation, whereas the hybrid regulation allows them to choose their regulation ex-post, that is, after observing their actual costs. Given that the welfare effects of the latter type of regulation depend strongly on the distribution of the random cost shocks $\theta$, concerning which we have made no assumptions, it is not possible to formally derive general results with regard to the preferability of either regulation concept. Therefore we will only provide a general discussion here and then support it with a numerical

\footnotetext{
${ }^{5}$ It is easily possible to see this formally by assuming that there would be a regulation that could separate the firms into two isolated permit markets and two different tax regimes with switching points $\beta_{\text {crit }_{1}}, \beta_{\text {crit }_{2}}, \beta_{\text {crit }_{3}} \in[\hat{\beta}-\Delta, \hat{\beta}+\Delta]$. Calculating the expected social costs as in (14), but with four integrals, and minimizing these with regard to $\beta_{\text {crit }_{1}}, \beta_{\text {crit }_{2}}, \beta_{\text {crit }_{3}}$ shows that the only solution with $\beta_{\text {crit }_{1}}, \beta_{\text {crit }_{2}}, \beta_{\text {crit }_{3}} \in[\hat{\beta}-\Delta, \hat{\beta}+\Delta]$ is a solution in which the firms are separated into two groups with the policy outlined in Proposition 11. This holds regardless of which instrument is applied to which subregion of $[\hat{\beta}-\Delta, \hat{\beta}+\Delta]$.
} 
example in the following section.

The main difference between the hybrid regulation and our concept is that the hybrid regulation allows to differentiate the regulation among different values of the random shocks. If the actual abatement costs are extremely high or low, a price-based regulation in the form of fixed taxes and subsidies is used, whereas for actual abatement costs close to their expected value, a permit market is used. This reduces the social costs of the uncertainty. However, all firms will always choose the same instrument; if the observed permit price falls between the subsidy and the tax, all firms participate in permit trading; otherwise they pay the tax or sell permits to the lower guarantee price. Thus the hybrid regulation does not differentiate among different types of firms.

Our approach has an opposite focus. The firms have to commit to a regulation before knowing their actual abatement costs. Thus the regulation cannot attenuate the social costs of the uncertainty. But the firms choose according to their technology, so that we achieve a differentiation according to firm types. As [17] has shown, whether a price- or a quantity-based regulation should be used, depends on the technology of firms. Thus differentiating the regulation with regard to firm types yields welfare benefits. Our concept realizes these benefits.

Given this discussion, it seems natural to expect that the hybrid regulation of [11] will be preferable if there is a high level of uncertainty (i.e., a large value of $\sigma^{2}$ ) but not too much technological heterogeneity (i.e., a small value of $\Delta$ ). In contrast, our approach should be preferable under conditions of considerable technological heterogeneity but not too large cost uncertainty. Given that it is impossible to gain an explicit description of the welfare effects of the hybrid regulation, even for simplistic distributional assumptions, we cannot prove this conjecture. However, it is intuitive and the numerical example in the following section supports it.

\section{A Detailed Example}

In this section we provide a numerical example to highlight the important aspects of our approach and to compare it to a single instrument policy as well as to the hybrid regulation of [11]. For this, we have chosen numerical values for the model parameters (see Table 1) that depict a case with substantial uncertainty and some firm heterogeneity. We have chosen $\varrho=1$ to facilitate the comparison of our approach with [11], where a single random variable influences the aggregate abatement costs. 


\begin{tabular}{|c|c|c|c|c|c|}
\hline & $\begin{array}{c}\delta=0.7 \\
z_{q}^{a u c}=0.05 \\
\end{array}$ & $\begin{array}{c}\delta=0.8 \\
z_{q}^{a u c}=0.05\end{array}$ & $\begin{array}{c}\delta=0.9 \\
z_{q}^{a u c}=0.05 \\
\end{array}$ & $\begin{array}{c}\delta=0.8 \\
z_{q}^{a u c}=0\end{array}$ & $\begin{array}{c}\delta=0.8 \\
z_{q}^{a u c}=\omega\end{array}$ \\
\hline \multicolumn{6}{|l|}{ Policy menu } \\
\hline$p_{p}$ & 1.733 & 1.625 & 1.529 & 1.625 & 1.625 \\
\hline$z_{q}+z_{q}^{a u c}$ & 1.226 & 1.320 & 1.404 & 1.320 & 1.320 \\
\hline$z_{p}$ & 0.947 & 1.022 & 1.087 & 1.072 & 0 \\
\hline$\beta_{c r i t}^{*}$ & 0.794 & 0.806 & 0.819 & 0.806 & 0.806 \\
\hline$z_{q}$ & 1.176 & 1.270 & 1.354 & 1.320 & 0.248 \\
\hline $\mathcal{E}\left(p_{q}\right)$ & 1.733 & 1.625 & 1.529 & 1.625 & 1.625 \\
\hline $\mathcal{E}(E)$ & 1.213 & 1.300 & 1.376 & 1.300 & 1.300 \\
\hline \multicolumn{6}{|l|}{ Only tax } \\
\hline $\begin{array}{l}p_{p} \\
\text { Only permit trading }\end{array}$ & 1.733 & 1.625 & 1.529 & & \\
\hline$z_{q}$ & 1.213 & 1.300 & 1.376 & & \\
\hline $\mathcal{E}\left(p_{q}\right)$ & 1.733 & 1.625 & 1.529 & & \\
\hline $\begin{array}{l}\text { Reduction in exp. soc. costs } \\
\text { compared to tax }\end{array}$ & $20.359 \%$ & $19.512 \%$ & $19.796 \%$ & & \\
\hline $\begin{array}{l}\text { Reduction in exp. soc. costs } \\
\text { compared to permits }\end{array}$ & $13.393 \%$ & $19.512 \%$ & $28.977 \%$ & & \\
\hline
\end{tabular}

Table 1: Design of the optimal policy menu and comparison with the single policy case in a numerical example.

The first four lines of Table 1 describe the optimal menu of policies as characterized in Proposition 1 for different values of $\delta$ and for different numbers of auctioned permits. They show that the tax always equals the expected permit price and that both are independent of $z_{q}^{a u c}$ but decreasing in $\delta$. The tax exemption is a decreasing function of $z_{q}^{a u c}$. Furthermore, $\beta_{c r i t}^{*}$ increases with $\delta$. This dependency is depicted in Figure 4, which also shows that, in this example, the expected social costs are very sensitive with regard to $\beta_{c r i t}^{*}$. So this example highlights the points of Proposition 1 and Corollary 2 .

The lower part of Table 1 compares the policy menu with a policy based only on a tax or only on permit trading. It shows that the expected emissions and the tax rate of our approach equal the optimal choices in the single policy case. However, by letting firms choose their regulation, our approach reduces the expected social costs of the regulation considerably (about $20 \%$ in this example). 


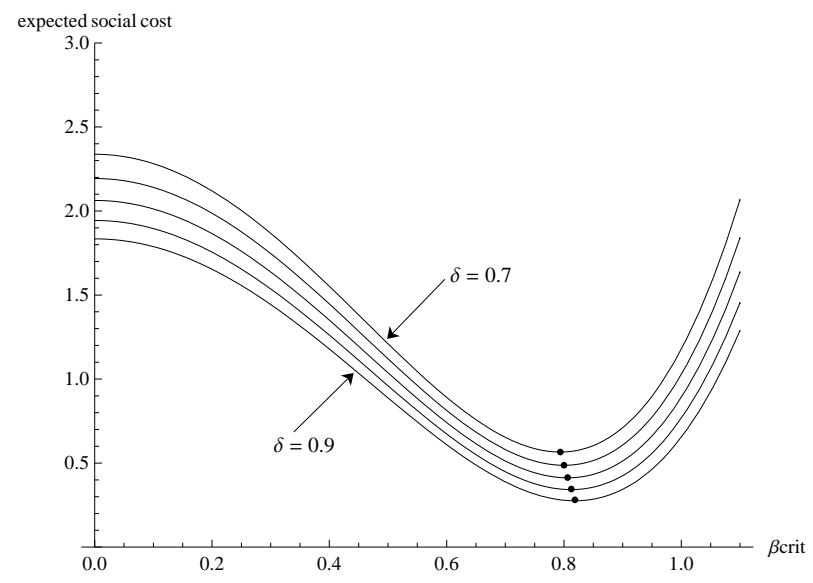

Figure 4: Expected social costs as a function of $\beta_{\text {crit }}$ for different values of $\delta$ $(\delta=0.70, \delta=0.75, \delta=0.80, \delta=0.85, \delta=0.90)$.

Finally, we compare our concept to the hybrid regulation advanced in [11]. For this, we assume that the uncertain cost shocks are uniformly distributed. To facilitate the comparison, we only use a tax and permit trading as instruments in the hybrid regulation, that is, in contrast to [11], there is no subsidy that establishes a lower boundary to the permit price. Figure 5 depicts the preferability of either approach for different values of $\sigma$, which corresponds to uncertainty, and $\Delta$, which corresponds to asymmetric information. As this figure shows, neither approach is always recommendable. The hybrid regulation performs better whenever there is much uncertainty and not too much asymmetric information. Our approach is recommendable in cases where asymmetric information is more important than uncertainty. This is in line with the conjecture made in the preceding section.

\section{Conclusions}

In this paper, we have considered a form of environmental policy that allows firms to choose among different regulation options. The intention of this device is to elicit private information about a firm's abatement costs in similarity to contractbased screening devices used on labor or insurance markets. We have shown that under uncertainty and asymmetric information, such a policy menu allows to achieve lower expected social costs than a single instrument. Thereby, the optimal policy menu is relatively simple to devise and thus provides an applicable solution 


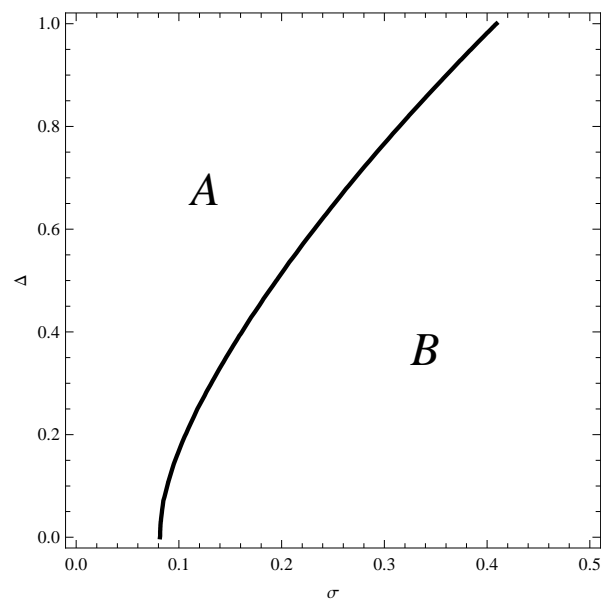

Figure 5: Comparison of our approach and the hybrid regulation for different levels of uncertainty $(\sigma)$ and asymmetric information $(\Delta)$. In region $A$ the policy menu is preferable, in region $\mathrm{B}$, the hybrid regulation is more recommendable.

to the problems posed by informational constraints in environmental policy. In addition, the optimal policy menu has some interesting theoretical properties. It is asymmetric with regard to choice between the price and quantity options in that a pooling equilibrium where all firms choose the tax can be optimal, whereas a pooling equilibrium with all firms choosing permit trading is never optimal. Also, rather surprisingly, the prices-versus-quantities criteria of [17] and [18] do not characterize to the firm that is indifferent between the policy options.

Our analysis extends the existing literature by focusing on alleviating the consequences of asymmetric information instead of those of uncertainty. Closest to our approach is the hybrid regulation scheme advanced in [11]. This scheme reduces the impact of uncertainty by using a price-based or a quantity-based regulation in dependency on the realization of random variables, like future prices. We use a price or a quantity regulation in dependency on the technological characteristics of a firm. Since firms differ with regard to these characteristics, price and quantity instruments will often be used simultaneously in our concept, whereas they are only used successively in [11].

As we have discussed and shown in a numerical example, both approaches can be advantageous with the hybrid regulation scheme being preferable in cases of strong cost uncertainty and not too strong firm heterogeneity and our approach being advisable in the opposite setup. Thus both concepts are complements that 
should be applied in different circumstances. Furthermore, it might be interesting to combine these approaches, that is, to let firms choose between a tax and a permit market with price limits. But this would results in a regulation that is most likely too complex for being implemented.

Our analysis is also connected to the screening literature, especially to the literature on insurance contracts. It shows that the main idea of eliciting private information by providing several options can be successfully transferred to the design of environmental policy. However, several adjustments are necessary. Most importantly, environmental policy is designed to balance the policy's effects on the environment with its effect on the regulated firms. Thus, in contrast to the screening literature, the costs of the screened individuals are part of the policy objective and not only a constraint. Also, there is neither competition among contract offers nor an exit-option in the policy setting, so that important constraints of the screening literature are missing.

Our investigation has been inspired by the climate policy recently implemented in Switzerland. This policy consists of an emission tax combined with a permit market and the regulated firms are allowed to choose between these options. There are two main differences between our model and this implemented policy. First, the firms that have chosen to participate in emissions trading in Switzerland have the option to rescind their choice at the end of a five year trading period. Given that they will lose all permits and will have to repay the taxes, it is unlikely that this option will be exercised. Furthermore, as shown in [5], different policy instruments will induce different technological choices and therefore firms are likely to become locked into their choice of policy. So our model neglects only a negligible point. Second, the policy in Switzerland does not include a tax exemption or auctioned permits. Our analysis shows that this is very likely suboptimal, because some firms will choose emission trading that should be regulated with a tax according to social welfare considerations. This theoretical result seems to apply in practice. So far, many firms have opted for permit trading. Given that the costs of emission volatility is rather small in the context of climate change due to the long retention period of greenhouse gases in the atmosphere, this seems to be suboptimal $]^{6}$

Altogether, our analysis shows that it is socially beneficial to let firms choose their regulation. It has long been accepted in economics that it is more efficient if firms decide upon their abatement activities under the incentives set by a tax

\footnotetext{
${ }^{6}$ See [8] for a detailed argumentation concerning the choice between price- and quantity-based regulation concepts for stock pollutants.
} 
or a permit price than if a governmental agency prescribed these activities in the context of a command-and-control policy. The reason is that firms are usually better informed about the costs of their abatement activities than a regulator. Our approach shows that this idea can be taken a step further: It is better if firms decide how they are regulated than if this decision is made by the government. Again, the firms simply have the better information and thus the government should only set the options from which the firms can choose.

\section{References}

1. R. A. Collinge and W. E. Oates, Efficiency in pollution control in the short and long runs: A system of rental emission permits, Canadian Journal of Economics 15, 178-188 (1982).

2. C. Henry, Microeconomics for Public Policy, Clarendon Press, Oxford (1989).

3. L. Karp and J. Livernois, Using automatic tax changes to control pollution emissions, Journal of Environmental Economics and Management 27, 38-48 (1994).

4. F. C. Krysiak, Ex-post efficient permit markets: A detailed analysis, Environmental and Resource Economics (forthcoming).

5. F. C. Krysiak, Prices vs. quantities: The effects on technology choice, Journal of Public Economics (forthcoming).

6. G. Meran and U. Schwalbe, Pollution control and collective penalties, Journal of Institutional and Theoretical Economics 143, 616-629 (1987).

7. A. A. Moledina, J. S. Coggins, S. Polasky, and C. Costello, Dynamic environmental policy with strategic firms: Prices versus quantities, Journal of Environmental Economics and Management 45, 356-376 (2003).

8. R. G. Newell and W. A. Pizer, Regulating stock externalities under uncertainty, Journal of Environmental Economics and Management 45, 416-432 (2003).

9. R. G. Newell, W. A. Pizer, and J. Zhang, Managing permit markets to stabilize prices, Environmental and Resource Economics 31, 133-157 (2005). 
10. J. G. Riley, Silver signals: Twenty-five years of screening and signaling, Journal of Economic Literature 39, 432-479 (2001).

11. M. J. Roberts and M. Spence, Effluent charges and licenses under uncertainty, Journal of Public Economics 5, 193-208 (1976).

12. M. Rothschild and J. E. Stiglitz, Equilibrium in competitive insurance markets: An essay on the economics of imperfect information, Quarterly Journal of Economics 90, 629-649 (1976).

13. K. Segerson, Uncertainty and incentives for nonpoint pollution control, Journal of Environmental Economics and Management 15, 87-98 (1988).

14. J. E. Stiglitz, The theory of screening, education, and the distribution of income, American Economic Review 65, 283-300 (1975).

15. J. E. Stiglitz, Monopoly, non-linear pricing and imperfect information: The insurance market, Review of Economic Studies 44, 407-430 (1977).

16. W. Unold and T. Requate, Pollution control by options trading, Economics Letters 73, 353-358 (2001).

17. M. L. Weitzman, Prices vs. quantities, Review of Economic Studies 41, 477491 (1974).

18. R. C. Williams III, Prices vs. quantities vs. tradable quantities, Working paper 9283, NBER (2002). 